\title{
Adaptation du métabolisme énergétique des ruminants à la sous-alimentation. Quantification au niveau de l'animal entier et de tissus corporels
}

\author{
I Ortigues
} INRA, laboratoire de la lactation et de l'élevage des ruminants,
Theix, 63122 Saint-Genès-Champanelle, France

(Reçu le 19 mars 1991; accepté le 28 août 1991)

\begin{abstract}
Résumé - Les ruminants répondent à une sous-alimentation en s'adaptant progressivement jusqu'à ce que, vraisemblablement, un nouvel équilibre s'instaure entre les apports alimentaires et les besoins. La diminution des besoins d'entretien qui en résulte provient d'une réduction du métabolisme de base, variable en fonction du niveau et de la durée de sous-alimentation. Ces modifications peuvent s'expliquer non seulement par des variations de poids vif, mais aussi par des changements au niveau de chaque tissu corporel (poids, métabolisme). Ceux-ci contribuent aux dépenses énergétiques de l'animal entier de façon non proportionnelle à leur poids. Ainsi, le tractus digestif, le foie et les masses musculaires contribuent pour $5-11,1-2,5$ et $35-50 \%$ du poids vif, mais pour $16-29$, 17-31 et environ $16 \%$ des dépenses énergétiques totales de l'animal, du fait d'une activité métabolique très différente entre tissus. La sous-alimentation conduit à une diminution de la contribution des viscères aux dépenses totales; les effets sur les masses musculaires sont moins connus. Ces variations de contribution proviennent de modifications de la masse respective des différents tissus, de leur approvisionnement en nutriments (diminution des débits sanguins en particulier) et/ou de leur activité métabolique.
\end{abstract}

métabolisme énergétique / sous-alimentation / adaptation / tissu corporel

Summary - Adaptation of energy metabolism to under-nutrition in ruminants. Quantification in whole animal and in indlvidual body tissues. Ruminants respond to underfeeding by a progressive adaptation until a new equilibrium between dietary supply and requirements is reached. The resulting drop in maintenance energy requirements originates from a decrease in basal metabolism that depends on the level and duration of undernutrition. These modifications are not totally accounted for by changes in live weight but might be explained by differential changes in individual tissue weight and metabolism. Tissues contribute to whole animal expenditure in a disproportionate manner relative to their weight. Thus digestive tract, liver and muscle masses contribute 5-11, 1-2.5 and $35-50 \%$ to live weight but about 16-29, 17-31 and about 16\% to whole animal energy expenditure, respectively, because of differences in tissue metabolic activity. Underfeeding results in a decrease in the contribution of viscera to energy expenditure, whereas results on muscle mass are scarce. These changes in contribution are due to changes in tissue mass, nutrient supply (blood flow) and/or in metabolic activity. 


\section{INTRODUCTION}

Lorsque les conditions économiques ou climatiques de production sont difficiles, il est de pratique courante, en nutrition animale, de réserver les aliments de meilleure qualité ou la majorité des stocks fourragers aux animaux de rapport (à l'engrais ou laitiers) aux dépens des animaux à faibles besoins tels que les vaches allaitantes. Pour ces derniers, il est fréquent de chercher à réduire au maximum leur coût de production, essentiellement durant la période hivernale où les disponibilités fourragères sont réduites. Ceci revient à limiter les apports alimentaires. Cette situation de sous-alimentation temporaire, utilisée traditionnellement comme technique d'élevage, risque de se développer avec l'extensification des productions animales.

Les conséquences de la sousalimentation chez les vaches allaitantes dépendent, d'une part de l'intensité de la sous-alimentation et d'autre part de la fonction physiologique considérée. Les stades physiologiques qui sont ainsi touchés par la sous-alimentation sont le milieu et la fin de la gestation ainsi que le début de la lactation. Si elle est appliquée pendant la gestation, la sous-alimentation ne menace pas le développement du veau, à moins que la vache ne perde du poids, ce qui est rarement le cas en France. Pendant la lactation, la sousalimentation n'a que peu d'effets sur la production laitière sauf si cette sousalimentation est de longue durée. Par contre, dans les troupeaux allaitants, la conséquence la plus importante de la sous-alimentation concerne l'aptitude des animaux à se reproduire. Ainsi, si la sousalimentation conduit à un état d'amaigrissement trop poussé, la venue en chaleurs des vaches est retardée et la fertilité (fécondation, survie embryonnaire) réduite (Petit, 1988). Il existe donc des limites à la sous-alimentation qui dépendent des fonc- tions physiologiques considérées. Ainsi donc, sont soulevées les questions suivantes:

- quels sont les besoins minimaux des animaux à faibles besoins ?

- de combien peut-on sous-alimenter un animal sans pénaliser sa production et sans hypothéquer sa carrière ? En d'autres termes, quelle est la capacité d'adaptation des animaux, et

- quelles sont leurs réponses à la sousalimentation?

Dans le contexte de l'élevage des vaches allaitantes, la sous-alimentation s'exprime d'abord en termes d'apports énergétiques totaux, avant de s'exprimer en termes d'apports azotés ou minéraux. Or des essais d'alimentation ont montré que, dans ces conditions, un animal s'adaptait et que sa perte de poids était plus faible que ce que l'on pouvait prévoir (Petit et Micol, 1981). Chez les vaches allaitantes, les besoins énergétiques d'entretien représentent environ les $2 / 3$ des besoins énergétiques totaux de l'animal sur une moyenne annuelle (Petit, 1988). Dans les essais d'alimentation, si l'on attribue le phénomène d'adaptation des animaux à la sous-alimentation uniquement à une réduction des besoins énergétiques d'entretien, celle-ci serait de l'ordre de 10-15\% par rapport aux besoins théoriques (Petit et Micol, 1981).

Cette revue se propose d'établir les bases physiologiques de l'adaptation du métabolisme énergétique des ruminants à la sous-alimentation. Elle s'attachera à quantifier cette adaptation au niveau de l'animal entier ou des tissus. Seuls, les effets sur les besoins énergétiques d'entretien seront développés vue leur prépondérance dans les besoins totaux. Ce travail concerne en priorité les vaches de type allaitant, mais rapporte des données obtenues sur d'autres types de ruminants en l'absence de résultats pertinents chez les vaches. 


\section{BESOINS ÉNERGÉTIQUES D'ENTRETIEN ET ADAPTATION}

\section{Besoins énergétiques d'entretien}

Les besoins énergétiques d'entretien sont définis par la quantité d'énergie ingérée par un animal qui n'entraîne ni gain ni perte d'énergie corporelle; la quantité d'énergie métabolisable ingérée (EM, calculée comme la différence entre l'énergie brute ingérée et la somme d'énergies fécale, urinaire et dégagée sous forme de méthane) est alors égale à la production de chaleur de cet animal. Les besoins d'entretien sont calculés, soit à partir de la production de chaleur d'un animal à jeun ou en bilan énergétique nul, soit à partir des rations qui n'entraînent pas de changement de poids vif à long terme. Ce dernier mode d'estimation, souvent utilisé dans les essais d'alimentation, doit être considéré avec précaution lorsqu'il ne s'accompagne pas de mesures précises de composition corporelle.

La plage de variation des besoins d'entretien s'étend de 80 à $160 \mathrm{kcal} E M / \mathrm{kg}$ $\mathrm{P}^{0,75}$ (poids métabolique) chez des bovins de race à viande (Johnson, 1984), selon les conditions de milieu (ex : climat, alimentation, activité physique), la composition corporelle (ex: viscères, rapport tissus protéiques/adipeux), l'état physiologique (ex : âge, lactation) et la variabilité interindividuelle. Cette dernière, de l'ordre de 5$10 \%$ (coefficient de variation, van Es, 1972), n'explique qu'une faible partie de la variation totale. Par contre, le niveau d'alimentation pourrait modifier les besoins d'entretien jusqu'à 40\% (Johnson, 1984). L'étude de l'adaptation à la sousalimentation s'intègre donc dans celle, plus générale, des facteurs de variation des besoins énergétiques d'entretien des animaux.

\section{Adaptation}

L'adaptation se définit comme un ensemble d'ajustements, mis en jeu au sein de l'organisme, qui tendent à minimiser les changements provoqués par des stimuli extérieurs (Beaton, 1985). Les caractéristiques des phénomènes d'adaptation sont les suivantes (Waterlow, 1985):

- l'adaptation est constituée d'un ensemble de différents mécanismes,

- son but est de maintenir un état stable dans l'organisme,

- un état stable n'est pas absolu mais est réalisé dans certaines limites préférentielles (intervalle de confiance),

- les mécanismes d'adaptation sont réversibles en cas de changement de l'environnement.

Les états stables sont caractérisés soit par l'homéostasie, c'est-à-dire le maintien de l'état interne de l'organisme dans des conditions stables (ex : glycémie), soit par l'énantiostasie, c'est-à-dire le maintien de fonctions physiologiques (ex : reproduction), (Hochachka et Somero, 1984). Cette distinction s'apparente à celle faite en nutrition animale entre la fonction d'entretien et celles de production.

Par conséquent, les mécanismes d'adaptation développés ci-dessous concernent les situations d'entretien ou d'homéostasie où l'organisme, non productif, va chercher à compenser un bilan énergétique négatif, créé par la sousalimentation. Dans ce contexte, on distingue un ensemble de réponses à la sousalimentation :

- une diminution du poids vif jusqu'à ce qu'un nouvel équilibre soit atteint,

- une modification de la composition corporelle, avec mobilisation des tissus corporels et modifications de la composition de la masse maigre corporelle, 
- des diminutions de l'activité métabolique des tissus ou organes,

- une modification de la digestibilité des régimes,

- une modification de l'activité physique des animaux.

Cette revue décrira les conséquences de la sous-alimentation sur l'animal entier (poids vif, composition corporelle) avant de développer une des composantes du calcul des besoins énergétiques : les dépenses énergétiques. Les effets de la sous-alimentation seront alors abordés au niveau de l'animal entier et des différents tissus corporels. Par contre, cette revue n'inclut pas les aspects de digestibilité, autre composante du calcul des besoins énergétiques, ni ceux du comportement animal.

\section{DIMINUTION DU POIDS VIF ET MOBILISATION DES RÉSERVES CORPORELLES, EFFETS DU TEMPS}

\section{Diminution du poids vif et mobilisation des réserves corporelles}

Le phénomène de diminution du poids vif des animaux sous-alimentés est bien connu et correspond à 2 mécanismes principaux :

- des variations de poids des contenus digestifs,

- des variations de la masse et de la composition corporelle.

Ces aspects ont été revus par Chilliard et al (1987) et Agabriel et Petit (1987). Ainsi, chez les vaches sous-alimentées, la perte de poids vif s'explique, pour environ les $2 / 3$, par une diminution de la masse des contenus digestifs. $\grave{A}$ court terme (1 semaine), une baisse de $1 \mathrm{~kg}$ de la matière sèche ingérée journalière se traduit par une variation de poids vif de $6 \mathrm{~kg}$, dont $4 \mathrm{~kg}$ de contenu du reticulo-rumen (Petit et Micol, 1981; Agabriel et Giraud, 1988). À plus long terme, cependant, les variations de poids des contenus digestifs en fonction du niveau alimentaire sont plus faibles (ARC, 1980; Agabriel et Petit, 1987; Chilliard et al, 1987). Le reste de la perte de poids vif correspond à une mobilisation des tissus corporels. Chez les bovins adultes la composition chimique des tissus mobilisés a été mesurée ou estimée (Chilliard et al, 1987). Cette dernière dépend d'abord de l'état d'engraissement initial de l'animal. Une vache Charolaise, adulte, sous-alimentée et de note d'état corporel élevée ( 3 à 4 ), présenterait une perte de masse corporelle composée essentiellement de lipides; par contre, une vache en mauvais état corporel (notée de 1 à 2) aurait une perte de masse corporelle composée de $20 \%$ de lipides, $16 \%$ de protéines et $64 \%$ d'eau et de minéraux (valeurs estimées par Agabriel et Petit, 1987).

\section{Evolution du poids vif en fonction du temps}

Un aspect important de ces observations qui a souvent été sous-estimé, concerne la progression de ces variations au cours du temps (adaptation, Clapperton et Blaxter, 1965; Parks, 1982). II semblerait en effet que pour un animal à l'entretien, dans un état d'équilibre donné, toute modification du niveau alimentaire entraîne des variations du poids vif, des contenus digestifs et de la masse corporelle qui se stabilisent progressivement avec le temps jusqu'à ce qu'un nouvel état d'équilibre entre les apports alimentaires et les besoins des animaux soit atteint (Turner et Taylor, 1983). La nécessité de prendre en compte l'effet du temps pour déterminer les besoins alimentaires des animaux a clairement été démontrée par Ledger et Sayers (1977). 
Ainsi, des bouvillons en croissance ont été maintenus à poids constant par une diminution des apports alimentaires de manière exponentielle au cours du temps. Après 6 mois, les quantités d'aliments nécessaires aux besoins d'entretien s'étaient stabilisées, selon la race et le poids vif, à seulement $50-66 \%$ des besoins théoriques estimés d'après ARC (1965) (fig 1). Un tel effet du temps sur les besoins d'entretien a aussi été noté par Meyer et Clawson (1964) et Burrin et al (1990).

Ces observations concernant l'effet du temps sur les réponses des animaux ont été systématisées par Turner et Taylor (1983). Ces auteurs ont calculé que les $2 / 3$ seulement de la réponse totale étaient obtenus au bout de 4 semaines, ce qui indique l'existence d'effets rémanents prolongés des niveaux alimentaires précédents. Ce phénomène pourrait avoir des conséquences non-négligeables sur l'estimation des besoins alimentaires des animaux. Par exemple, cet effet du temps expliquerait, selon eux, une partie des différences obte- nues (Waldo et Tyrrell, 1980) dans les bilans énergétiques (et donc, dans les calculs des besoins énergétiques) obtenus par des essais d'alimentation (adaptation à long terme) ou par des mesures en chambres respiratoires (adaptation à court terme). Pour une quantité d'énergie digestible ingérée similaire (environ $62 \mathrm{KJ} / \mathrm{j}$ ), la quantité d'énergie retenue a été de $5,6 \mathrm{MJ} / \mathrm{j}$ dans l'essai d'alimentation et de $9,4 \mathrm{MJ} / \mathrm{j}$ en chambres respiratoires.

\section{Évolution de la mobllisation des réserves corporelles en fonction du temps}

L'effet du temps est important, non seulement en termes de poids vif, mais aussi de composition corporelle. Ce problème a été étudié chez les rats (Goodman et al, 1980) et les pingouins (Chérel et Le Maho, 1985; Robin et al, 1988), mais n'est pas connu chez les ruminants. Les pingouins jeûnent naturellement 4-5 mois/an, pendant leur

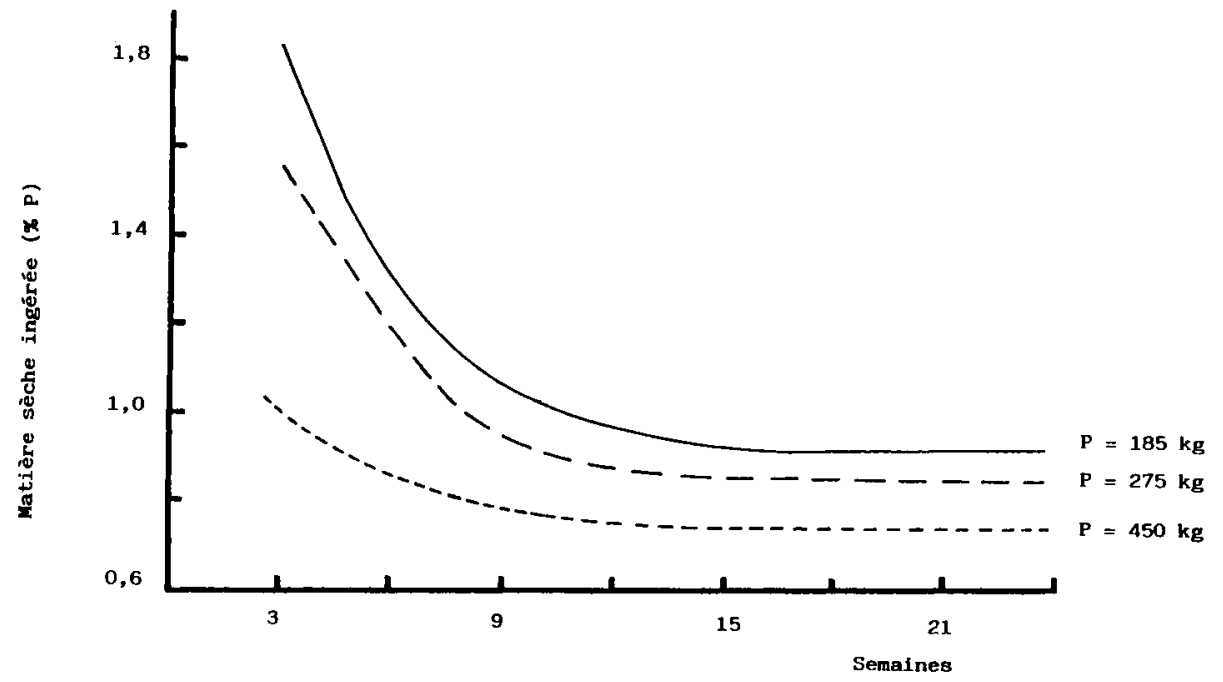

Fig 1. Variations des apports alimentaires au cours du temps, chez des bouvillons en croissance maintenus à poids (P) constant (Ledger et Sayers, 1977). Après 6 mois d'expérience, les besoins d'entretien d'animaux pesant 185, 275 ou $450 \mathrm{~kg}$ correspondaient respectivement à $50 \%, 54 \%$ ou $64 \%$ de leurs besoins théoriques. $-P=185 \mathrm{~kg} ;--P=275 \mathrm{~kg} ;---P=450 \mathrm{~kg}$. 
a) $\quad$ Ortigues

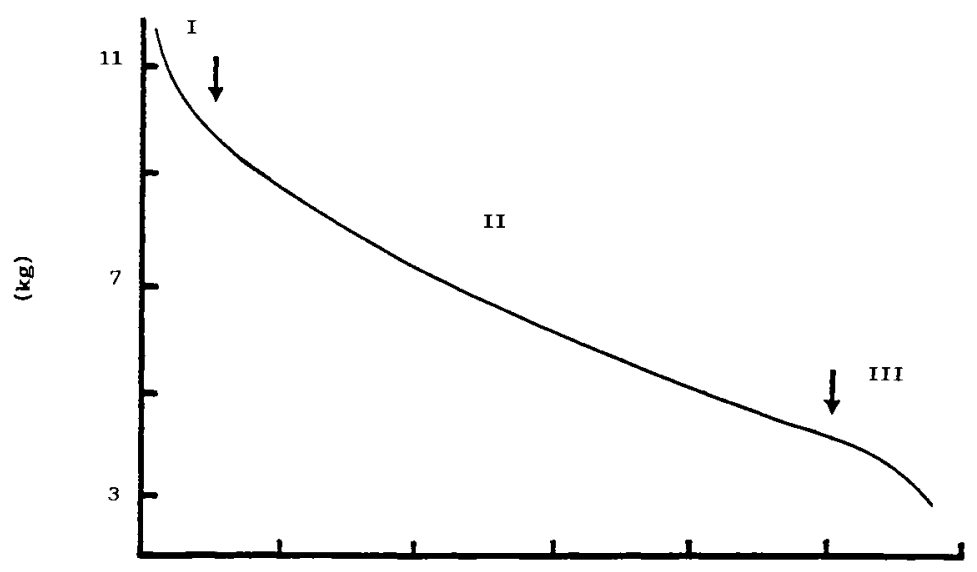

b)
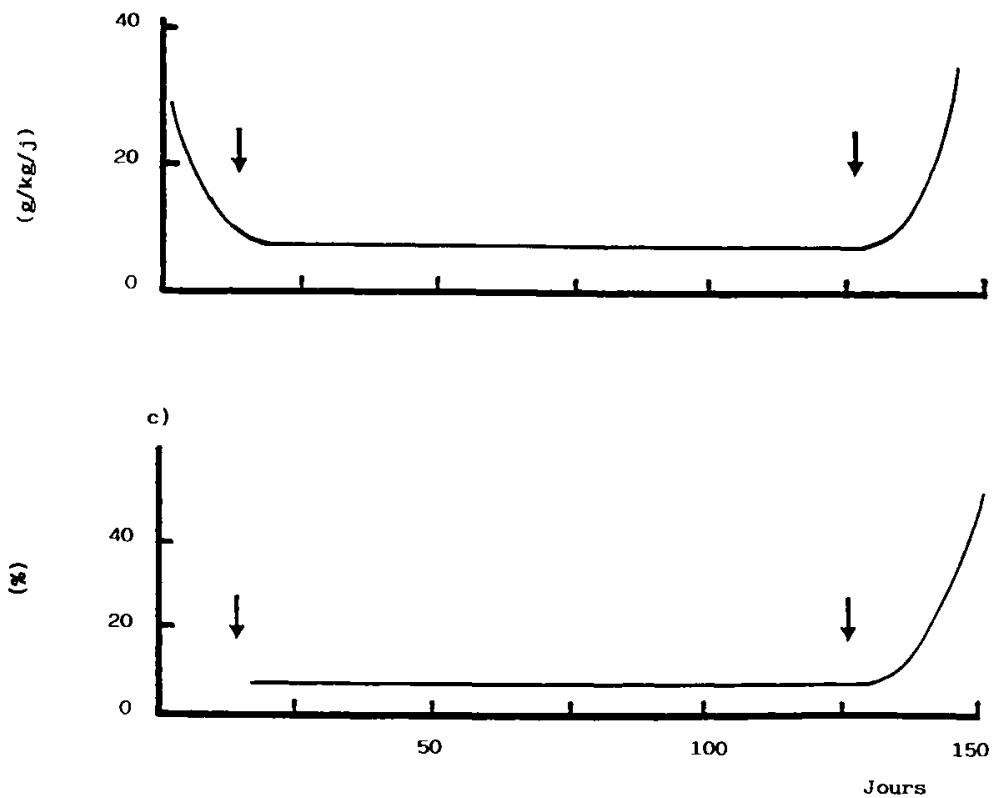

Fig 2. Variations de poids vif $(P)$ (a), de la vitesse relative de la perte de poids [dP / (P.dt)] (b) et de la proportion d'énergie mobilisée sous forme de protéines (c) chez des pingouins au cours des phases I, II et Ill de jeûne (Chérel et Le Maho, 1985; Robin et al, 1988).

période de reproduction et d'élevage des jeunes. Ces auteurs ont montré que la perte de poids des pingouins pendant le jeûne s'effectuait en 3 étapes (fig 2). Pendant la première phase (2 semaines), la perte de poids des pingouins est très ra- 
pide mais diminue avec le temps. II n'y a malheureusement pas eu de mesures de composition corporelle durant cette période. La deuxième étape correspond à une phase de stabilisation où la perte relative de poids journalière reste constante (à $1 \%$ du poids vif) pendant 4 mois et où les protéines mobilisées ne représentent que $4 \%$ de l'énergie mobilisée. Une économie de protéines corporelles est donc réalisée pendant cette période. Durant la dernière étape (3-4 semaines), la perte de poids vif s'accélère; la proportion d'énergie mobilisée sous forme de protéines augmente, conduisant à la mort, alors que les réserves lipidiques des animaux restaient non négligeables (environ 10\% du poids vif contre $\mathbf{2 8 \%}$ initialement).

Chez les ruminants sous-alimentés (et non pas à jeun), il est probable que la phase I soit beaucoup plus longue et que les transitions entre les phases I, II et III soient mal caractérisées. Une telle détermination serait néanmoins nécessaire pour connaître la variation de composition des nutriments endogènes mobilisés ainsi que l'existence éventuelle d'une épargne azotée.

L'évolution du poids vif et de la composition corporelle des animaux sousalimentés pourraient donc influer, d'une part sur l'estimation des besoins et, d'autre part, sur la composition optimale des régimes à offrir à ces animaux.

\section{ADAPTATION DU MÉTABOLISME ÉNERGÉTIQUE}

Parallèlement à leur perte de poids, les animaux sous-alimentés diminuent leurs dépenses énergétiques. Ce phénomène d'économie d'énergie a été mis en évidence par des travaux déjà anciens. Marston (1948) a alimenté des moutons adultes pendant 2,5 mois à des niveaux égaux à $0,5,1,1,5$ ou 2 fois l'entretien. Le métabolisme au jeûne de ces animaux (rapporté au poids métabolique, $\mathrm{P} 0,75$ ) s'élevait à $248,284,288$ et $313 \mathrm{~kJ} . \mathrm{kg} P-0,75$. $\mathrm{j}^{-1}$, respectivement, ce qui correspondrait à une économie d'énergie réalisée par les animaux sous-alimentés et exprimée par unité de poids de l'ordre de $13-20 \%$ par rapport aux dépenses énergétiques d'animaux alimentés à 1 ou 2 fois l'entretien. Les travaux réalisés depuis confirment l'existence d'une économie d'énergie réalisée par l'organisme, à la suite d'une baisse du niveau alimentaire, mais ne permettent pas de quantifier cette économie de manière reproductible, ainsi que l'indiquent les résultats bibliographiques rapportés au tableau I ou par O'-Donovan (1984). La durée et l'importance de la sousalimentation, la race et la composition corporelle initiale des animaux peuvent expliquer la variabilité notée.

Chez les bovins à viande, il n'y a eu qu'un très petit nombre de mesures des dépenses énergétiques d'animaux à jeun, à la suite d'une sous-alimentation (tableau I). La plupart des données disponibles correspond à des résultats de besoins énergétiques d'entretien obtenus de manière indirecte en s'appuyant sur des modifications de poids vif et de composition corporelle mesurées dans des essais d'alimentation. En particulier, l'effet du niveau alimentaire a long terme sur les besoins d'entretien a été considéré comme étant similaire à l'effet de l'état d'engraissement des animaux sur leurs besoins d'entretien. Il est en effet estimé que l'état d'engraissement des animaux résulte du niveau alimentaire antérieur. Les résultats ainsi obtenus sont contradictoires et n'indiquent pas toujours des besoins d'entretien (exprimés par unité de poids) plus faibles pour les animaux sous-alimentés, donc amaigris (tableau II).

Par exemple, Ferrell et Jenkins (1984) ont maintenu des vaches, présentant différents états d'engraissement à poids cons- 
Tableau I. Variations de la production de chaleur à jeun (M) des ruminants en fonction de leur niveau alimentaire précédent.

\begin{tabular}{|c|c|c|c|c|}
\hline Références & Animal & $\begin{array}{l}\text { Niveau } \\
\text { alimentaire } \\
\text { précédent" }\end{array}$ & $\begin{array}{l}\text { Durée } \\
\text { (i) }\end{array}$ & $\begin{array}{c}M^{* *} \\
\left(k J . k g P-0,75, j^{-1}\right)\end{array}$ \\
\hline $\begin{array}{l}\text { Marston } \\
(1948)\end{array}$ & $\begin{array}{l}\text { brebis } \\
\text { adultes }\end{array}$ & $\begin{array}{l}0,5 \text { entretien } \\
1 \text { entretien } \\
1,5 \text { entretien } \\
2 \text { entretien }\end{array}$ & $\begin{array}{l}70 \\
70 \\
70 \\
70\end{array}$ & $\begin{array}{l}248 \\
284 \\
287 \\
313\end{array}$ \\
\hline $\begin{array}{l}\text { Farrell et al } \\
\text { (1972) }\end{array}$ & $\begin{array}{l}\text { brebis } \\
\text { adultes }\end{array}$ & $\begin{array}{l}\text { sous-alimentation } \\
\text { (pâturage) } \\
1 \text { entretien } \\
\text { (pâturage) }\end{array}$ & $\begin{array}{c}30 \\
180 \\
365 \\
30 \\
80 \\
365\end{array}$ & $\begin{array}{c}257 \\
215 \\
(301) \\
303 \\
280 \\
(258)\end{array}$ \\
\hline $\begin{array}{l}\text { Graham et al } \\
(1974)\end{array}$ & $\begin{array}{l}\text { moutons }<1 \text { an } \\
\text { moutons } 1 \text { an } \\
\text { moutons } 2 \text { ans }\end{array}$ & $\begin{array}{l}0,5 \text { ad libitum } \\
\text { ad libitum } \\
0,5 \text { ad libitum } \\
\text { ad libitum } \\
0,5 \text { ad libitum } \\
\text { ad libitum }\end{array}$ & $\begin{array}{l}21-321 \\
21-231 \\
378-665 \\
378-665 \\
714-812 \\
714-812\end{array}$ & $\begin{array}{l}264-302 \\
302-378 \\
237-297 \\
231-287 \\
247-254 \\
246-251\end{array}$ \\
\hline $\begin{array}{l}\text { Gingins et al } \\
(1980)\end{array}$ & $\begin{array}{l}\text { moutons } \\
\text { adultes }\end{array}$ & $\begin{array}{l}0,25-0,5 \\
\text { entretien } \\
1 \text { entretien }\end{array}$ & $\begin{array}{r}119 \\
28\end{array}$ & $\begin{array}{l}249 \\
279\end{array}$ \\
\hline $\begin{array}{l}\text { Koong et al } \\
\text { (1982) }\end{array}$ & $\begin{array}{l}\text { agneaux en } \\
\text { croissance }\end{array}$ & $\begin{array}{l}\text { bas }(\mathrm{GMQ}=-100 \mathrm{~g} / \mathrm{j}) \\
\text { moyen } \\
(\mathrm{GMQ}=100 \mathrm{~g} / \mathrm{j}) \\
\text { haut }(\mathrm{GMQ}=400 \mathrm{~g} / \mathrm{j})\end{array}$ & $\begin{array}{l}84 \\
84 \\
84\end{array}$ & $\begin{array}{l}290 \\
295 \\
372\end{array}$ \\
\hline $\begin{array}{l}\text { Silanikove } \\
\text { (1987) }\end{array}$ & $\begin{array}{l}\text { chèvres } \\
\text { adultes } \\
\text { (Bédouines) }\end{array}$ & $\begin{array}{l}0,45 \text { ad libitum } \\
0,65 \text { ad libitum } \\
\text { ad libitum }\end{array}$ & $\begin{array}{l}12 \\
12 \\
12\end{array}$ & $\begin{array}{l}140 \\
214 \\
320\end{array}$ \\
\hline $\begin{array}{l}\text { Frisch et Vercoe } \\
(1977)\end{array}$ & bouvillons & $\begin{array}{l}\text { foin de luzerne } \\
\text { - entretien } \\
\text { - ad libitum } \\
\text { foin de prairie } \\
\text { - entretien } \\
\text { - ad libitum }\end{array}$ & $\begin{array}{l}100 \\
100 \\
100 \\
100\end{array}$ & $\begin{array}{l}85 \mathrm{~kJ} \cdot \mathrm{kg} P^{-1} \cdot j^{-1} \\
97 \mathrm{~kJ} \cdot \mathrm{kg} \mathrm{P} P^{-1} \cdot j^{-1} \\
82 \mathrm{~kJ} \cdot \mathrm{kg} \cdot P^{-1} \cdot \mathrm{j}^{-1} \\
86 \mathrm{~kJ} \cdot \mathrm{kg} P^{-1} \cdot \mathrm{j}^{-1}\end{array}$ \\
\hline $\begin{array}{l}\text { Varga et Tyrrell } \\
\text { (1989) }\end{array}$ & $\begin{array}{l}\text { bouvillons } \\
\text { en } \\
\text { croissance }\end{array}$ & $\begin{array}{l}1,4 \text { entretien } \\
\text { suivi de } \\
\text { l'entretien }\end{array}$ & $\begin{array}{r}240 \\
42\end{array}$ & 380 \\
\hline & & $\begin{array}{l}2 \text { entretien } \\
\text { suivi de l'entretien }\end{array}$ & $\begin{array}{r}120 \\
42\end{array}$ & 347 \\
\hline
\end{tabular}

- GMQ : gain de poids vif moyen quotidien; *" les mesures de métabolisme au jeûne $(M)$ ont été réalisées sans que les animaux aient été alimentés à l'entretien dans les jours précédant le début du jeûne, sauf dans les travaux réalisés par Graham et al (1974). 
Tableau II. Variations des besoins d'entretien estimés de vaches adultes en fonction de leur état d'engraissement.

\begin{tabular}{|c|c|c|c|}
\hline Référence & Race & $\begin{array}{l}\text { Etat } \\
\text { d'engraissement }^{\text {tong }}\end{array}$ & $\begin{array}{l}\text { Besoins d'entretien } \\
\left(k J \text { EM.kg } P^{-0}, 75 . j^{1}\right)\end{array}$ \\
\hline $\begin{array}{l}\text { Thompson et al } \\
\text { (1983) }\end{array}$ & $\begin{array}{l}\text { Angus } \times \text { Hereford } \\
\text { Angus } \times \text { Holstein }\end{array}$ & $\begin{array}{r}10,3 \% \text { lipides * } \\
15,5 \% \text { lipides } \\
7,9 \% \text { lipides } \\
12,3 \% \text { lipides }\end{array}$ & $\begin{array}{l}550 \\
517 \\
580 \\
596\end{array}$ \\
\hline $\begin{array}{l}\text { Russel et Wright } \\
\text { (1983) }\end{array}$ & $\begin{array}{l}\text { Hereford x Frison } \\
\text { Shorthorn x Galloway } \\
400 \mathrm{~kg} \\
600 \mathrm{~kg}\end{array}$ & $\begin{array}{l}\text { note }=1 / 5^{\star \star} \\
\text { note }=3 / 5 \\
\text { note }=1 / 5 \\
\text { note }=3 / 5\end{array}$ & $\begin{array}{l}586 \\
443 \\
648 \\
490\end{array}$ \\
\hline $\begin{array}{l}\text { Ferrell et Jenkins } \\
\text { (1984) }\end{array}$ & $\begin{array}{l}\text { Angus } \\
\text { Hereford } \\
\text { Simmental }\end{array}$ & $\begin{array}{l}\text { maigre } \\
\text { grasse } \\
\text { maigre } \\
\text { grasse } \\
\text { maigre } \\
\text { grasse }\end{array}$ & $\begin{array}{l}362 \\
625 \\
414 \\
596 \\
485 \\
634\end{array}$ \\
\hline $\begin{array}{l}\text { Wagner et al } \\
\text { (1988) }\end{array}$ & Hereford & $\begin{array}{l}\text { note }=3 / 10 \\
\text { note }=5 / 10 \\
\text { note }=7 / 10\end{array}$ & $\begin{array}{l}632 \\
657 \\
602\end{array}$ \\
\hline
\end{tabular}

" Exprimé par rapport au poids vif; ** Note d'état corporel.

tant et ont mesuré leurs variations d'énergie corporelle (à l'aide de biopsies de tissus adipeux au niveau de la $12^{\theta}$ côte). Ils ont effectivement obtenu des besoins d'entretien plus faibles pour les vaches maigres que pour les vaches grasses (362, 414 et 485 contre 625,596 et $634 \mathrm{~kJ}$ EM.kg P-0.75. $\mathrm{j}^{-1}$ pour les races Angus, Hereford et Simmental respectivement). Par contre, Russel et Wright (1983) ont calculé, par régression, à partir des variations de poids d'animaux dans des états d'engraissement différents et alimentés à des niveaux allant de 0,5 à 1,2 fois l'entretien, des besoins plus élevés pour les vaches maigres que pour des vaches grasses (648 contre 490 kJ EM.kg P-0,75.j-1, pour une vache de $600 \mathrm{~kg}$ de note d'état 1 ou 3 respectivement). Ces derniers résultats confortaient ceux de Klosterman et al (1968) et Hohenboken et al (1972). Enfin, les travaux de Wagner et al (1988) semblaient indiquer une relation curvilinéaire entre l'état d'engraissement des vaches de race à viande et leurs besoins d'entretien hivernaux. Ces derniers augmentaient avec l'état d'engraissement jusqu'au stade où les dépôts adipeux sous-cutanés permettant une isolation thermique, faisaient alors baisser les besoins d'entretien. 
Ces résultats contradictoires obtenus sur bovins restent discutables en raison de l'imprécision des mesures réalisées pour estimer les besoins d'entretien et la composition corporelle. II est à noter que plusieurs auteurs (tableau III) n'ont trouvé aucune différence significative entre le métabolisme au jeûne de moutons à différents états d'engraissement, mesuré en chambres respiratoires, bien que les animaux maigres aient tendance à avoir des dépenses énergétiques plus élevées.

Pour expliquer ces divergences de résultats, il a été proposé (Webster, 1981) de tenir compte de la composition corporelle des animaux et, en particulier, d'exprimer la production de chaleur (ou les besoins d'entretien) par rapport à la masse maigre. En effet, les différents constituants corporels (essentiellement protéines et graisse) présentent des taux de synthèse et des taux de renouvellement très diffé- rents, ce qui se traduit par des coûts énergétiques d'entretien beaucoup plus élevés pour la masse maigre que pour les tissus adipeux. Néanmoins, la masse maigre n'est pas de composition ni de métabolisme homogènes. II faut donc prendre en compte chacun des compartiments de la masse maigre, ainsi que cela est développé ci-dessous.

\section{RÉPONSES DES DIFFÉRENTS TISSUS CORPORELS A UNE BAISSE DU NIVEAU ALIMENTAIRE}

Les résultats rapportés ci-dessous sont une compilation des données disponibles sur ruminants; la majorité a été obtenue sur des animaux en croissance et sur des périodes relativement courtes (mesures in vivo).

Tableau III. Variations de la production de chaleur à jeun (M) de moutons adultes en fonction de leur état d'engraissement.

\begin{tabular}{|c|c|c|c|}
\hline Référence & Etat corporel ${ }^{*}$ & $\begin{array}{l}\text { Niveau } \\
\text { alimentaire } \\
\text { précédent }\end{array}$ & $\stackrel{M}{M}$ \\
\hline Graham (1964) & $\begin{array}{l}\text { maigre } \\
\text { moyen } \\
\text { gras }\end{array}$ & $\begin{array}{l}900 \mathrm{~g} \text { foin } \mathrm{j} \\
\text { pendant } \\
3 \text { semaines }\end{array}$ & $\begin{array}{l}251 \\
226 \\
205\end{array}$ \\
\hline Graham (1967) & $\begin{array}{l}\text { 7\% lipides dans PVV } \\
19 \% \text { lipides dans PVV } \\
29 \% \text { lipides dans PVV } \\
32 \% \text { lipides dans PVV }\end{array}$ & $\begin{array}{l}900 \mathrm{~g} \text { foin } / \mathrm{j} \\
\text { pendant } \\
\mathrm{j}-2 \\
\text { semaines }\end{array}$ & $\begin{array}{l}221 \\
214 \\
217 \\
214\end{array}$ \\
\hline Graham (1969) & $\begin{array}{l}\text { 10\% lipides dans PVV } \\
33 \% \text { lipides dans PVV }\end{array}$ & $\begin{array}{l}700 \mathrm{~g} \text { foin } / \mathrm{j} \\
\text { pendant } 3 \mathrm{j}\end{array}$ & $\begin{array}{l}236 \\
217\end{array}$ \\
\hline McNiven (1984) & $\begin{array}{l}\text { maigre }(54 \mathrm{~kg}) \\
\text { moyen }(63 \mathrm{~kg}) \\
\text { gras }(82 \mathrm{~kg})\end{array}$ & $\begin{array}{l}900 \mathrm{~g} \text { foin } / \mathrm{j} \\
\text { pendant } \\
2 \text { semaines }\end{array}$ & $\begin{array}{l}325 \\
313 \\
301\end{array}$ \\
\hline
\end{tabular}

- PVV : poids vif vide. 


\section{Variations de la contribution des tissus aux dépenses énergétiques totales}

De manière générale, la contribution des differents tissus ou organes aux dépenses de l'organisme entier n'est pas proportionnelle à leur masse. Ainsi, un exercice de modélisation sur agneaux en croissance réunissant de nombreux résultats bibliographiques présente les estimations les plus complètes, disponibles à nos jours, des contributions des différents tissus aux dépenses énergétiques de l'animal entier (Gill et al, 1989). Ces contributions s'élèveraient à $20,0 \%$ pour le foie, $19,2 \%$ pour le tube digestif, $18,0 \%$ pour la peau, $16,4 \%$ pour les muscles ainsi qu'à $9,9 \%$ pour le tissu adipeux, $8,9 \%$ pour les glandes salivaires et pancréatiques, $5,2 \%$ pour le cœur, $4,6 \%$ pour le système nerveux central, $3,7 \%$ pour le système réticuloendothélial et $2,1 \%$ pour les reins. Ces estimations soulignent l'importance des tissus protéiques et, en particulier, du tractus digestif, du foie et de la peau dans le dépenses énergétiques totales de l'animal, malgré leur faible importance pondérale : environ $5-11,1-2,5$ et $6-9 \%$ du poids vif (tableau V). Par comparaison, la contribution des masses musculaires aux dépenses totales est sensiblement équivalente $(16 \%)$ bien que les muscles représentent $35-50 \%$ du poids vif. Des estimations similaires ont été données par plusieurs auteurs (Canas et al, 1982; Koong et al, 1985; Ma et Foster, 1986; Huntington and Reynolds, 1987). Du fait de la prépondérence du tube digestif, du foie et des masses musculaires dans les dépenses énergétiques de l'animal entier, la suite de cette revue se limitera à ces tissus.

Lors d'une baisse du niveau alimentaire, les dépenses énergétiques du tube digestif, du foie et des masses musculaires, mesurées in vivo, diminuent presque propor- tionnellement aux dépenses énergétiques de l'animal entier. II existe cependant une tendance selon laquelle cette baisse serait plus importante au niveau du tube digestif et du foie.

Les dépenses énergétiques du tube digestif, exprimées par unité de poids métabolique de l'animal entier, varient de $3,1-3,4$ à 8,3-7,2 kJ.kg P-0,75. $\mathrm{h}^{-1}$ entre des animaux à jeun ou ingérant $1006-1808 \mathrm{~kJ}$ EM.kg P-0.75. . -1 $^{-1}$ (tableau IV). Webster et al (1975) ont fait état d'une relation curvilinéaire croissante entre l'énergie métabolisable ingérée et les dépenses énergétiques du tube digestif, indiquant que ces dernières augmentaient de $4,7 \mathrm{~kJ} \cdot \mathrm{h}^{-1} \cdot \mathrm{MJ}-1$ EMI entre 0 et 1 fois le niveau d'entretien, mais de $9,9 \mathrm{~kJ} \cdot \mathrm{h}^{-1} \cdot \mathrm{MJ}^{-1} \mathrm{EMI}$ entre 1 et 2 fois le niveau d'entretien. Les données disponibles dans la littérature sont en nombre insuffisant pour confirmer une telle réponse. La contribution du tube digestif aux dépenses énergétiques de l'animal entier oscille donc entre 16 et $29 \%$, augmentant légèrement (mais pas toujours significativement) avec le niveau alimentaire. Par contre, cette augmentation serait nettement plus marquée pour des régimes riches en fourrages (Reynolds et Tyrrell, 1989; Eisemann et Nienaber, 1990).

Au niveau du foie, les dépenses énergétiques de l'organe, rapportées au poids métabolique de l'animal, sont du même ordre de grandeur que celles du tube digestif $\left(3,9-8,9 \mathrm{~kJ} . \mathrm{kg} \mathrm{P}^{-0,75} \cdot \mathrm{h}^{-1}\right)$ et varient de manière similaire, plus rapide (Burrin et al, 1989) ou moins rapide (Eisemann et Nienaber, 1990) avec le niveau alimentaire (tableau IV). La contribution du foie aux dépenses de l'animal entier varie ainsi entre 17 et $31 \%$. II est intéressant de noter que chez des animaux à jeun, le foie conservant une activité métabolique importante pourrait contribuer aux dépenses énergétiques totales de manière plus importante que chez l'animal nourri (Eisemann et Nienaber, 1980). 
Tableau IV. Variations des dépenses énergétiques du tube digestif et du foie rapportées au poids métabolique (DE, $\mathrm{kJ} \mathrm{h}^{-1} . \mathrm{kg} \mathrm{P}-0,75$ ) et aux dépenses énergétiques totales (DE/DET, \%) de l'animal entier en fonction du niveau alimentaire $\left(\mathrm{KJ} \mathrm{EM}_{\mathrm{j}} \mathrm{j}^{-1} . \mathrm{kg} \mathrm{P}-0,75\right)$.

\begin{tabular}{|c|c|c|c|c|c|c|}
\hline \multirow[t]{2}{*}{ Référence } & \multirow[t]{2}{*}{ Animal } & \multirow{2}{*}{$\begin{array}{l}\text { Niveau } \\
\text { alimentaire }\end{array}$} & \multicolumn{2}{|c|}{ Tube digestif } & \multicolumn{2}{|c|}{ Foir } \\
\hline & & & $D E$ & $D E / D E T$ & $D E$ & $D E / D E T$ \\
\hline $\begin{array}{l}\text { Huntington } \\
\text { et Tyrrell } \\
\text { (1985) }\end{array}$ & $\begin{array}{l}\text { vaches } \\
\text { laitières }\end{array}$ & $\begin{array}{l}1732 \\
1808\end{array}$ & $\begin{array}{l}7,91 \\
7,22\end{array}$ & $\begin{array}{l}16,7 \\
18,5\end{array}$ & & \\
\hline $\begin{array}{l}\text { Reynolds } \\
\text { et Tyrrell } \\
(1987)\end{array}$ & génisses & $\begin{array}{r}70^{*} \\
130^{*}\end{array}$ & & $\begin{array}{l}25,1 \\
27,6\end{array}$ & & $\begin{array}{l}17,2 \\
21,8\end{array}$ \\
\hline $\begin{array}{l}\text { Burrin } \\
\text { et al } \\
(1989)\end{array}$ & agneaux & $\begin{array}{l}391 \\
656 \\
725 \\
818\end{array}$ & $\begin{array}{l}3,80 \\
5,35 \\
5,66 \\
5,16\end{array}$ & $\begin{array}{l}19,0 \\
24,0 \\
25,0 \\
25,0\end{array}$ & $\begin{array}{l}3,90 \\
5,38 \\
7,00 \\
8,93\end{array}$ & $\begin{array}{l}22,0 \\
25,0 \\
31,0 \\
44,0\end{array}$ \\
\hline $\begin{array}{l}\text { Eisemann } \\
\text { et Nienaber } \\
(1990)\end{array}$ & bouvillons & $\begin{array}{r}0 \\
818 \\
0 \\
1007\end{array}$ & $\begin{array}{l}3,06 \\
5,09 \\
3,41 \\
6,74\end{array}$ & $\begin{array}{l}17,8 \\
19,9 \\
19,9 \\
25,4\end{array}$ & $\begin{array}{l}4,37 \\
5,23 \\
4,54 \\
5,46\end{array}$ & $\begin{array}{l}25,4 \\
20,4 \\
26,5 \\
20,6\end{array}$ \\
\hline $\begin{array}{l}\text { Huntington } \\
\text { et al } \\
\text { (1988) }\end{array}$ & bouvillons & $\begin{array}{r}793 \\
1098 \\
715 \\
877\end{array}$ & $\begin{array}{l}6,95 \\
8,72 \\
6,72 \\
8,72\end{array}$ & $\begin{array}{l}23,9 \\
23,4 \\
25,1 \\
29,3\end{array}$ & & \\
\hline $\begin{array}{l}\text { Reynolds } \\
\text { et Tyrrell } \\
\text { (1989) }\end{array}$ & génisses & $\begin{array}{r}601 \\
1006 \\
500 \\
1009\end{array}$ & $\begin{array}{l}5,67 \\
8,26 \\
4,71 \\
6,68\end{array}$ & $\begin{array}{l}25,8 \\
27,6 \\
23,8 \\
22,6\end{array}$ & $\begin{array}{l}4,46 \\
7,43 \\
3,85 \\
7,22\end{array}$ & $\begin{array}{l}18,5 \\
26,3 \\
20,6 \\
21,6\end{array}$ \\
\hline
\end{tabular}

* Exprimé en g matière sèche.kg $\mathrm{P}^{-0,75} \cdot \mathrm{j}^{-1}$.

Au niveau musculaire, moins de données sont disponibles et elles n'ont pas été rapportées à la masse musculaire totale. II n'a pas été montré de variations postprandiales des dépenses énergétiques du train arrière (Christopherson et Brockman, 1989). Le jeûne entraîne une diminution des dépenses de la masse musculaire mais ne modifie pas la contri- bution de ces tissus à celles de l'animal entier, sauf au froid (Bell et Thompson, 1979; Eisemann et Nienaber, 1990). Par contre dans une expérience sur agneaux en croissance, Harris et al (1989) ont mesuré que la patte arrière augmentait de manière significative sa contribution aux dépenses énergétiques totales de 4,1 à $5,4 \%$ alors que le niveau alimentaire pas- 
sait de 1,8 à 0,6 fois le niveau d'entretien. En fait, il avait été observé que les moutons au bas niveau alimentaire étaient plus énervés et passaient plus de temps debout que les autres. Il est à noter que les expériences qui ont montré une diminution du métabolisme à jeun lors de sousalimentation n'ont jamais pris en compte le degré d'activité de l'animal alors qu'il peut introduire un biais considérable dans les résultats (JM Brockway, comm pers).

Ces variations des dépenses énergétiques des tissus mesurées in vivo à la suite d'un changement du niveau alimentaire, peuvent résulter de variations de la masse des tissus, ou de leur approvisionnement en oxygène, du fait de modifications dans les débits sanguins ou dans leur activité métabolique (captation d'oxygène par unité de masse). Ces 3 points sont développés ci-dessous.

\section{Variations de poids \\ des tissus corporels}

Du fait de l'importance des organes internes dans le métabolisme énergétique, la réduction des dépenses (exprimée par unité de poids vif) consécutive à une baisse du niveau alimentaire, a été attribuée en grande partie à ceux-ci (Huntington et Reynolds, 1987; Johnson et al, 1990). De même, les variations des besoins énergétiques d'entretien observées entre races ou stades physiologiques proviendraient de différences relatives dans le poids des organes digestifs (Jenkins et al,

1986). Ces résultats sont basés essentiellement sur des corrélations positives entre les variations du métabolisme à jeun et le poids des organes digestifs. Une telle relation a aussi été obtenue entre des animaux ayant le même poids vif final mais ayant subi des niveaux alimentaires différents (Koong et al, 1985). Ainsi, par exemple, chez des porcs maintenus à poids constant $(50 \mathrm{~kg})$ pendant $66 \mathrm{j}$ par une baisse progressive des apports alimentaires, il y a eu une diminution tout à fait parallèle du métabolisme à jeun de ces animaux et de la taille des organes digestifs (Koon et Nienaber, 1985). Des corrélations similaires ont été obtenues essentiellement chez des animaux en croissance (Johnson et al, 1985; Ferrell et Koong, 1986; Ferrell et al, 1986; Burrin et al, 1990) : le foie, les estomacs et les intestins sont les tissus dont le poids répond le plus rapidement aux variations de niveau alimentaire (Seebeck, 1967). II a été calculé qu'en moyenne le poids du foie et du tube digestif augmentait d'environ 14 et $28 \mathrm{~g} / \mathrm{kg}$ po,75 lorsque le niveau alimentaire passait de 1 à 2 fois le niveau d'entretien (Johnson et al, 1990).

Cependant, les variations de poids des tissus digestifs en fonction du niveau alimentaire mesurés sur animaux adultes (Doreau et al, 1985; Robelin et al, 1990) ou à long terme (High et Barton, 1965; Leche, 1973; Varga et Tyrrell, 1989) sont moins systématiques (tableau $V$ ), ce qui suggère que les modifications de la masse des tissus digestifs ne suffisent pas à expliquer la diminution des dépenses énergétiques avec le niveau alimentaire ( $\mathrm{Ma}$ et Foster, 1986). Ainsi, Rompala et al (1988) ont fait varier de manière significative la masse des estomacs $(+6 \%)$ et du gros intestin $(+13 \%)$ de moutons, alimentés de manière iso-énergétique, en modifiant l'encombrement de la ration avec de la poudre de polyéthylène. Ces variations ne se sont pas traduites par des changements du métabolisme à jeun de ces animaux.

\section{Variations des débits sanguins avec le niveau alimentaire}

De façon générale, des études de physiologie comparée entre espèces animales in- 
Tableau V. Poids des tissus digestifs (exprimé en pourcentage du poids vif vide) chez des ruminants adultes en fonction du niveau d'alimentation.

\begin{tabular}{|c|c|c|c|c|c|c|c|}
\hline Référence & Animal & $\begin{array}{l}\text { Niveau } \\
\text { alimentaire * }\end{array}$ & Durée & Rumen & Intestins & Foie & $\begin{array}{l}\text { Tube } \\
\text { digestif }\end{array}$ \\
\hline $\begin{array}{l}\text { Hight et } \\
\text { Barton } \\
(1965)^{* *}\end{array}$ & brebis & $\begin{array}{l}\text { abattage } \\
\text { GMQ }=150 \mathrm{~g} \\
\mathrm{GMQ}=150 \mathrm{~g} \\
\mathrm{GMQ}=150 \mathrm{~g} \\
\text { puis ad libitum }\end{array}$ & $\begin{array}{r}\text { initial } \\
21 j \\
42 j \\
21 j \\
38 j\end{array}$ & & & $\begin{array}{l}2,48 \\
2,00 \\
2,32 \\
2,53\end{array}$ & $\begin{array}{r}11,05 \\
8,69 \\
9,62 \\
10,17\end{array}$ \\
\hline $\begin{array}{l}\text { Keenan } \\
\text { ot al } \\
\text { (1969) }\end{array}$ & $\begin{array}{l}\text { moutons } \\
\text { adultes }\end{array}$ & $\begin{array}{l}0,33 \text { entretien } \\
0,46 \text { entretien } \\
\text { entretien }\end{array}$ & $\begin{array}{l}28 j \\
84 j \\
84 j\end{array}$ & & & $\begin{array}{l}1,09 \\
1,74 \\
1,98\end{array}$ & \\
\hline \multirow{3}{*}{$\begin{array}{l}\text { Ferrell et } \\
\text { Jenkins } \\
(1984)\end{array}$} & $\begin{array}{l}\text { vaches } \\
\text { taries }\end{array}$ & $\begin{array}{l}469 \mathrm{~kJ} \text { EM.kg } \\
\mathrm{P}-0,75, \mathrm{j}^{-1}\end{array}$ & $140 j$ & & & 0,96 & \\
\hline & non-gestantes & $\begin{array}{l}745 \mathrm{~kJ} \text { EM.kg } \\
P-0,75 . j^{-1}\end{array}$ & $140 j$ & & & 1,07 & \\
\hline & & ad libitum & $140 \mathrm{j}$ & & & 1,11 & \\
\hline $\begin{array}{l}\text { Doreau } \\
\text { et } a l \\
\text { (1985) }\end{array}$ & $\begin{array}{l}\text { vaches } \\
\text { laitières } \\
\text { - taries, } \\
\text { non-gestantes } \\
\text { en lactation }\end{array}$ & $\begin{array}{l}7,0 \mathrm{~kg} \mathrm{MSl} / \mathrm{j} \\
11,8 \mathrm{~kg} \mathrm{MSl} / \mathrm{j} \\
13,5 \mathrm{~kg} \mathrm{MSl} / \mathrm{j} \\
15,0 \mathrm{~kg} \mathrm{MSl} / \mathrm{j}\end{array}$ & $\begin{array}{l}150 j \\
150 j \\
150 j \\
150 j\end{array}$ & $\begin{array}{l}2,92 \\
2,26 \\
2,16 \\
2,68\end{array}$ & $\begin{array}{l}3,21 \\
2,77 \\
2,72 \\
3,21\end{array}$ & & \\
\hline $\begin{array}{l}\text { Jenkins } \\
\text { et } a l \\
(1986)\end{array}$ & $\begin{array}{l}\text { vaches à } \\
\text { viande, } \\
\text { taries, } \\
\text { non-gestantes }\end{array}$ & $\begin{array}{l}\text { entretien } \\
\text { ad libitum }\end{array}$ & $\begin{array}{l}84 j \\
84 j\end{array}$ & & & $\begin{array}{l}0,94 \\
1,04\end{array}$ & $\begin{array}{r}9,16 \\
11,64\end{array}$ \\
\hline \multirow[t]{4}{*}{$\begin{array}{l}\text { Robelin } \\
\text { et al } \\
(1990)^{\star \star \star}\end{array}$} & $\begin{array}{l}\text { vaches } \\
\text { taries, } \\
\text { non gestantes }\end{array}$ & & & & & & \\
\hline & Holstein & $\begin{array}{l}6 \mathrm{~kg} \mathrm{MSI} / \mathrm{j} \\
\text { suivi de } 12,7 \mathrm{~kg} \\
\text { MSI/j }\end{array}$ & $\begin{array}{r}21 j \\
153 j\end{array}$ & $\begin{array}{l}2,92 \\
2,43\end{array}$ & $\begin{array}{l}3,23 \\
2,76\end{array}$ & $\begin{array}{l}1,73 \\
1,55\end{array}$ & $\begin{array}{l}8,07 \\
6,95\end{array}$ \\
\hline & Limousin & $\begin{array}{l}6 \mathrm{~kg} \mathrm{MSl} / \mathrm{j} \\
\text { suivi de } 9,7 \mathrm{~kg} \\
\mathrm{MSI} / \mathrm{j}\end{array}$ & $\begin{array}{r}21 j \\
123 j\end{array}$ & $\begin{array}{l}2,26 \\
1,81\end{array}$ & $\begin{array}{l}2,10 \\
2,03\end{array}$ & $\begin{array}{l}1,24 \\
1,08\end{array}$ & $\begin{array}{l}5,89 \\
4,97\end{array}$ \\
\hline & Charolais & $\begin{array}{l}6 \mathrm{~kg} \mathrm{MSI} / \mathrm{j} \\
\text { suivi de } 14,7 \mathrm{~kg} \\
\mathrm{MSI} / \mathrm{j}\end{array}$ & $\begin{array}{r}21 j \mathfrak{j} \\
133 j\end{array}$ & $\begin{array}{l}2,34 \\
2,25\end{array}$ & $\begin{array}{l}2,54 \\
2,66\end{array}$ & $\begin{array}{l}1,29 \\
1,04\end{array}$ & $\begin{array}{l}6,36 \\
6,14\end{array}$ \\
\hline
\end{tabular}

" GMQ : gain de poids vil moyen quotidien; EM : énergie métabolisable; MSI : matière sèche ingérée; "* les poids du foie et du tube digestif sont rapportés au poids de carcasse; '** les quantités d'aliment distribuées durant la période expérimentale correspondent à $90 \%$ de la capacité d'ingestion de chaque race.

diquent que les variations de débit sanguin sont les principaux facteurs de contrôle de l'intensité métabolique (Coulson, 1986).
Diverses expériences ont montré que la sous-alimentation entraînait une baisse du volume plasmatique total (Lederman et 
Rosso, 1989) et du débit cardiaque total (Ahokas et al, 1983; Sakanashi et al, 1987). Ce dernier est réduit proportionnellement à la perte de poids vif de l'animal et peut s'accompagner d'une certaine redistribution, sous contrôle hormonal, du débit sanguin à travers les différents tissus (Ahokas et al, 1983; Ma et Foster, 1986; Sakanashi et al, 1987). Par exemple chez le rat, 5 j de jeûne modifient les proportions du débit sanguin total irrigant le foie (de 2,0 à 3,3\%), le tractus digestif (de 17,7 à $15,9 \%$ ) et la peau (de 11,5 à $6,8 \%)(\mathrm{Ma}$ et Foster, 1986). Chez les ruminants, la majorité des données disponibles sur les variations de débit sanguin avec le niveau alimentaire concernent l'irrigation du tube digestif et du foie, plutôt que l'irrigation musculaire. Malheureusement, elles ne sont pas exprimées par unité de poids des tissus considérés alors qu'elles sont sans doute très liées aux variations de poids de ceux-ci.

Ainsi, au niveau splanchnique, plusieurs auteurs ont montré que le débit sanguin porte (DSP) variait avec le niveau alimentaire des animaux (ex : Lomax et Baird, 1983). Cette relation positive serait (tableau VI) soit linéaire (Huntington, 1984; Wieghart et al, 1986), soit curvilinéaire (Webster et al, 1975). Un regroupement de données bibliographiques, obtenues sur ovins et bovins et exprimées par $\mathrm{kg} d e$ poids métabolique, a permis de confirmer ces relations et surtout d'établir des rela-

Tableau VI. Relations entre les débits sanguins porte (P) et sus-hépatique (SH) et le niveau d'alimentation chez les ruminants.

\begin{tabular}{|c|c|c|c|}
\hline Référence & Relation * & $r$ & Sources \\
\hline $\begin{array}{l}\text { Webster } \\
\text { et al (1975) }\end{array}$ & $\begin{array}{l}P=1,326+0,554 e^{0,0978 \mathrm{EM}} \\
(\mathrm{I} / \mathrm{min})\end{array}$ & - & $\begin{array}{l}\text { données } \\
\text { propres }(n=43)\end{array}$ \\
\hline $\begin{array}{l}\text { Huntington } \\
(1984)\end{array}$ & $\begin{array}{l}P=5,867+0,09667 \mathrm{EM} \\
(1 / \mathrm{min})\end{array}$ & 0,75 & $\begin{array}{l}\text { données } \\
\text { propres }(n=59)\end{array}$ \\
\hline $\begin{array}{l}\text { Wiegart } \\
\text { et al } \\
(1986)\end{array}$ & $\begin{array}{l}P=33,514+1,1297 \mathrm{ED} \\
(\mathrm{l} / \mathrm{min})\end{array}$ & 0,87 & $\begin{array}{l}\text { données propres } \\
\text { et } \\
\text { bibliographiques } \\
(n=24)\end{array}$ \\
\hline $\begin{array}{l}\text { Cet } \\
\text { article }\end{array}$ & $\begin{array}{l}P=81,392+0,0666 \mathrm{EM} \\
\left(\mathrm{ml}^{\mathrm{min}} \mathrm{n}^{-1} . \mathrm{kg} \mathrm{P}-0,75\right) \quad\left(\mathrm{kJ} . \mathrm{j}^{-1} \cdot \mathrm{kg} \mathrm{P}^{-0,75}\right)\end{array}$ & 0,71 & $\begin{array}{l}\text { données } \\
\text { bibliographiques } \\
* *(n=34)\end{array}$ \\
\hline $\begin{array}{l}\text { Cet } \\
\text { article }\end{array}$ & $\begin{array}{l}\mathrm{SH}=91,883+0,0815 \mathrm{EM} \\
\left(\mathrm{ml}^{\mathrm{min}} \mathrm{min}^{-1} . \mathrm{kg} \mathrm{P}-0,75\right) \quad\left(\mathrm{kJ}_{\mathrm{j}} \mathrm{j}^{-1} \cdot \mathrm{kg} \mathrm{P} \mathrm{P}^{-0,75}\right)\end{array}$ & 0,96 & $\begin{array}{l}\text { données } \\
\text { bibliographiques } \\
* * *(n=16)\end{array}$ \\
\hline
\end{tabular}

\footnotetext{
"EM, ED : énergie digestible, métabolisable; "“ Heitman et al (1986); Huntington et al (1980, 1981, 1983); Huntington (1984); Huntington et Prior (1983); Prior et al (1981); ainsi que les réferences données en note "**; "** Bergman et Wolff (1971); Burrin et al (1989); Eisemann et Nienaber (1989); Heitman et Bergman (1981); Wieghart et al (1986).
} 
tions similaires pour le débit sanguin en veines sus-hépatiques. Malgré le nombre limité de résultats disponibles sur ruminants, il semblerait que les débits sanguins sus-hépatiques augmentent avec le niveau alimentaire et que cet effet résulte essentiellement de l'élévation des débits portes et d'une moindre mesure de celle des débits de l'artère hépatique (tableau VI). D'après les relations données au tableau $\mathrm{V} 1$, les débits des veines porte et sus-hépatique et de l'artère hépatique passent de 112,129 et 17 à 143,167 et 24 $\mathrm{ml} \mathrm{min}^{-1} \mathrm{~kg} \mathrm{P} \mathrm{P}^{-0,75}$ lorsque le niveau alimentaire passe de 1 à 2 fois celui de l'entretien. Ces variations de débit hépatique seraient, selon une étude, directement liées aux modifications de poids du foie (Burrin et al, 1989).

La majorité des études sur les mécanismes de régulation des débits sanguins splanchniques en fonction des quantités ingérées ont porté sur des effets à court terme, en particulier celui du repas. Au niveau du tube digestif, l'augmentation postprandiale des débits sanguins est un phénomène connu, résultant d'une accélération du rythme et du débit cardiaques, de modifications des résistances vasculaires (Chou, 1983; Barnes et al, 1986), d'une augmentation présumée du nombre de capillaires irrigués dans cette région (densité capillaire dépendant de la résistance des vaisseaux précapillaires, Shepherd, 1982; Granger et al, 1983) et d'une redistribution des volumes sanguins entre régions anatomiques aux dépens des membres et des tissus adipeux (Edelstone et Holzman, 1981; Chou, 1983; Barnes et al, 1986). Une hypothèse avancée par Shepherd (1982) suggère que le flux sanguin à travers ces tissus est régulé par leur activité métabolique. L'apport d'oxygène serait ainsi maintenu adéquat pour couvrir les besoins énergétiques nécessaires à la motilité intestinale (Chou et Gallavan, 1982), la sécrétion (Kaufman,
1982) et l'absorption des nutriments (Mailman, 1982). Ces mêmes auteurs soulignent cependant qu'il ne semble pas exister de relation de cause à effet simple entre ces différents facteurs.

Contrairement aux autres organes digestifs, le foie ne contrôle pas son débit sanguin en fonction de ses besoins métaboliques propres mais en fonction des besoins du reste de l'organisme (Lautt, 1983). La vascularisation hépatique ne contrôle pas le débit porte, qui dépend du tube digestif, mais régule le flux sanguin de l'artère hépatique de manière à maintenir constant le débit hépatique total. $\mathrm{Ce}$ mécanisme de réponse tampon de l'artère hépatique ne serait présent qu'à court terme ( $2 \mathrm{~h}$ au maximum) (Lautt, 1983).

Au niveau musculaire, le débit sanguin varie aussi avec le niveau alimentaire (Bird et al, 1981; Pethick et al, 1981; Early et al, 1984, 1987; Gregory et Christopherson, 1986; Eisemann et Nienaber, 1990) mais les données sont en nombre insuffisant pour établir une relation précise. Par contre, ces variations sont faibles par rapport à celles liées à l'activité physique. Ainsi, le débit sanguin en aorte postérieure a été majoré de $65 \%$ lorsque le niveau alimentaire est passé de 0 à 2,2 fois le niveau d'entretien (Eisemann et Nienaber, 1990) alors qu'il peut être jusqu'à quintuplé par l'activité physique (Jarret et al, 1976; Bird et al, 1981; Pethick et al, 1987).

A long terme, la vascularisation des tissus est modifiée en fonction de leur activité métabolique (Guyton, 1976). Cependant l'ensemble de ces résultats ne permet pas d'établir de relations quantitatives entre le niveau alimentaire, le poids de l'animal et des différents tissus, le volume sanguin, le débit cardiaque et le débit sanguin dans les différents tissus. Ces relations seraient utiles car la mesure du débit sanguin est délicate et présente un certain nombre de problèmes techniques qui peuvent être en 
grande partie responsables de la variabilité notée dans les mesures des dépenses énergétiques de tissus corporels particuliers.

\section{Variations de l'activité métabolique}

Les variations de poids des tissus et de débits sanguins avec le niveau alimentaire laissent supposer des modifications dans l'utilisation des nutriments par les tissus (telles que l'intensité des transports cellulaires et celles de la synthèse et de la dégradation protéique) et donc de l'activité métabolique de ceux-ci. Cette activité est appréciée par la consommation en oxygène du tissu exprimée par unité de poids et par unité de temps. La quasi-totalité de ces données a été obtenue in vitro après prélèvements de tissus par biopsies ou à l'abattage.

Chez les ruminants, la consommation d'oxygène de l'épithélium du tube digestif s'élève en moyenne à $4,6(3,5-5,4) \mathrm{nmol}$ $\mathrm{O}_{2} \cdot \mathrm{min}^{-1} \cdot \mathrm{mg}^{-1}$ tissu sec au niveau du rumen (Kelly et al, 1989; Burrin et al, 1990). Elle est de $8,2(2,1-16,8) \mathrm{nmol}$ $\mathrm{O}_{2} \cdot \mathrm{min}^{-1} \cdot \mathrm{mg}^{-1}$ tissu sec au niveau de l'intestin grêle (McBride et Milligan, 1982, 1984, 1985; Rompala et Hoagland, 1987; Rompala et al, 1987, 1988; McBride et al, 1989; Burrin et al, 1990) et de $6,8 \mathrm{nmol}$ $\mathrm{O}_{2} \cdot \mathrm{min}^{-1} \cdot \mathrm{mg}^{-1}$ tissu sec au niveau du colon (Rompala et al, 1988). Aucune étude réalisée jusqu'à présent n'a mis en évidence d'effet significatif du niveau alimentaire sur l'activité métabolique de l'épithélium du tube digestif, bien qu'une tendance apparaisse parfois à l'augmentation de cette activité avec le niveau alimentaire (tableau VII). II a, par contre, été noté une élévation de la consommation en $\mathrm{O}_{2}$ de l'épithélium ruminal $7 \mathrm{~h}$ après le repas (Kelly et al, 1989).

Au niveau du foie, la consommation en $\mathrm{O}_{2}$, mesurée in vitro, est plus faible que celle obtenue au niveau du tube digestif. Elle est de l'ordre de $3,3(1,7-5,6) \mathrm{nmol}$ $\mathrm{O}_{2} \cdot \mathrm{min}^{-1} \cdot \mathrm{mg}^{-1}$ tissu sec (tableau VII). La majorité des expérimentations n'ont, à nouveau, pas mis en évidence de variations significatives de l'activité métabolique du foie avec le niveau alimentaire (tableau VII) sauf celle de Ferrell et Koong (1985).

En ce qui concerne les muscles, il n'y a pas eu de mesures de leurs variations de consommation en oxygène en fonction du niveau alimentaire, publiées sur ruminants.

Ces études, qui sont pratiquement les seules disponibles sur ruminants à ce jour, présentent une forte variabilité, notamment entre équipes de recherche, et posent un problème d'interprétation liée à la méthodologie employée. En effet, les consommations moyennes $\mathrm{d}^{\prime} \mathrm{O}_{2}$ du foie de 3,3 nmol $\mathrm{O}_{2} \cdot \mathrm{min}^{-1} \cdot \mathrm{mg}^{-1}$ tissu sec ont été obtenues en utilisant des milieux d'incubation de type Krebs-Henseleit-Hepes alors que, sur rats, des valeurs de 15-16 nmol ont été mesurées par Burrin et al (1988) avec des milieux contenant des acides aminés et de l'insuline. Ces dernières sont tout à fait similaires aux consommations en $\mathrm{O}_{2}$ calculées à partir de résultats in vivo sur agneaux (Burrin et al, 1989; 1990), en supposant une teneur en matière sèche des tissus hépatiques de l'ordre de 0,30. D'autres facteurs tels que la teneur en oxygène du milieu d'incubation (McBride et Milligan, 1985), la préparation du tissu isolé (Umbreit et al, 1964) et l'apport de nutriments et d'hormones, influe sur sa consommation $\mathrm{d}^{\prime} \mathrm{O}_{2}$ (Lipinski, 1989; Schneider et al, 1990). Ces limites méthodologiques peuvent en partie expliquer le fait que les variations mesurées d'activité métabolique des tissus sont faibles, alors que de nombreuses données mettent en évidence une augmentation marquée dans l'intensité des composantes métaboliques des dépenses énergétiques, telles que le. turnover protéique, les transports actifs et les cycles de substrats avec le niveau alimentaire (Summers et al, 1988). 
Tableau VII. Effet du niveau alimentaire sur la consommation en oxygène $\left(\mathrm{nmol} \mathrm{O}_{2} \cdot \mathrm{min}^{-1} \cdot \mathrm{mg}^{-1}\right.$ tissu $\mathrm{sec}$ ) du foie, du rumen et de l'intestin grêle in vitro.

\begin{tabular}{|c|c|c|c|c|c|}
\hline \multirow[t]{2}{*}{ Référence } & \multirow[t]{2}{*}{ Animal } & \multirow[t]{2}{*}{ Niveau alimentaire * } & \multicolumn{3}{|c|}{ Consommation en $\mathrm{O}_{2}$} \\
\hline & & & Foie $* *$ & Rumen & $\begin{array}{l}\text { Intestin } \\
\text { grêle }\end{array}$ \\
\hline McBride et Milligan (1982) & moutons & $\begin{array}{l}\text { entretien } \\
\text { ad libitum }\end{array}$ & & & $\begin{array}{l}7,3 \\
6,9\end{array}$ \\
\hline McBride et Milligan (1985) & moutons & $\begin{array}{l}48 \mathrm{~h} \text { à jeun } \\
\text { entretien } \\
2 \times \text { entretien }\end{array}$ & & & $\begin{array}{l}5,2 \\
5,6 \\
6,1\end{array}$ \\
\hline Ferrell et Koong (1985) & agneaux & $\begin{array}{l}- \text { initial } \\
- \text { GMQ }=0 \mathrm{~kg}(6 \mathrm{sem}) \\
\text { puis } G M Q=0,4 \mathrm{~kg}(6 \mathrm{sem}) \\
\text { puis } G M Q=0 \mathrm{~kg}(6 \mathrm{sem}) \\
- \text { GMQ }=0,4 \mathrm{~kg}(6 \mathrm{sem}) \\
\text { puis } G M Q=0 \mathrm{~kg}(6 \mathrm{sem}) \\
\text { puis } G M Q=0 \mathrm{~kg}(6 \mathrm{sem})\end{array}$ & $\begin{array}{l}4,7 \\
4,6 \\
5,2 \\
3,1 \\
4,4 \\
4,0 \\
2,4\end{array}$ & & \\
\hline Rompala et Hoagland (1987) & moutons & $\begin{array}{l}40 \% \text { ad libitum } \\
\text { ad libitum }\end{array}$ & & & $\begin{array}{l}15,7 \\
14,7\end{array}$ \\
\hline Kelly et al (1989) & bouvillons & $\begin{array}{l}\text { O h après le repas } \\
3 \mathrm{~h} \text { après le repas } \\
7 \mathrm{~h} \text { après le repas }\end{array}$ & & $\begin{array}{l}5,0 \\
4,3 \\
5,4\end{array}$ & \\
\hline Burrin et al (1990) & agneaux & $\begin{array}{r}1087 \text { kJ EM.kg PVV-0,75 } \\
494 \text { kJ EM.kg PVV-0,75 } \\
1097 \text { kJ EM.kg PVV-0,75 } \\
1309 \text { kJ EM.kg PVV-0,75 }\end{array}$ & $\begin{array}{l}2,0 \\
2,6 \\
1,8 \\
2,7\end{array}$ & $\begin{array}{l}3,6 \\
4,4 \\
3,5 \\
3,8\end{array}$ & $\begin{array}{l}2,2 \\
2,1\end{array}$ \\
\hline
\end{tabular}

" $G M Q$ : gain de poids vif moyen quotidien; EM : énergie métabolisable; $P V V$ : poids vif vide; "* En supposant une teneur en matière sèche des tissus hépatiques égale à 0,27 (résultats non publiés).

\section{CONCLUSIONS}

L'adaptation du métabolisme énergétique à la sous-alimentation résulte de 2 mécanismes simultanés. D'une part, les dépenses énergétiques de l'animal à jeun (exprimées par unité de poids) baissent avec le niveau alimentaire, permettant une économie de 13-20\%. D'autre part, les be- soins énergétiques d'animaux maintenus à un niveau alimentaire bas, semblent diminuer de manière importante avec le temps (34-50\% en 6 mois).

Des études ont été réalisées pour déterminer si cette baisse des dépenses énergétiques d'entretien pouvait être attribuée à certains tissus corporels particuliers. Le tube digestif et le foie ont fait l'objet de la 
majorité de ces études car ils représentent une part importante des dépenses énergétiques totales ( 19 et $20 \%$, respectivement) malgré leur faible poids relatif. La réponse des 2 autres tissus, les muscles et la peau, qui contribuent aussi fortement aux dépenses (16 et $18 \%$ ) a été moins étudiée. Des corrélations significatives ont été obtenues sur animaux en croissance entre les variations des dépenses énergétiques en fonction du niveau alimentaire et le poids des tissus digestifs, imputant à ces derniers l'origine des économies d'énergie, mesurées sur l'animal entier. Ces relations sont moins systématiques sur animaux adultes. D'autre part, les mesures réalisées in vivo ne confirment pas cette hypothèse de manière aussi tranchée. En effet, les dépenses énergétiques du tube digestif, du foie et du muscle diminuent avec le niveau alimentaire, alors que leur contribution aux dépenses de l'animal entier ne semblent pas être fortement modifiées. La réduction des dépenses énergétiques de sites anatomiques particuliers mesurée in vivo est liée à des variations de débit sanguin plutôt qu'à des modifications mesurables d'activité métabolique de ces tissus.

Cet état des connaissances sur la quantification de l'adaptation des dépenses énergétiques d'entretien à la sousalimentation montre qu'un certain nombre de données doivent encore être obtenues avant de pouvoir être intégrées dans un ensemble utilisable à des fins pratiques pour l'alimentation des vaches allaitantes.

\section{RÉFÉRENCES}

Agabriel J, Giraud JM (1988) Contenu ruminal de la vache charolaise. Influence d'une brusque variation du niveau alimentaire. Reprod Nutr Dev 28, 107-108

Agabriel J, Petit M (1987) Recommandations alimentaires pour les vaches allaitantes. Bull Tech CRZV Theix, INRA 70, 153-166
Agricultural Research Council (1965) The Nutrient Requirements of Ruminant Livestock. No 2 Ruminants. Agric Res Council, Londres

Agricultural Research Council (1980) The Nutrient Requirements of Ruminant Livestock. Commonwealth Agricultural Bureaux, Farnham Royal, Slough

Ahokas PA, Anderson GD, Lipshitz J (1983) Effect of dietary restriction, during the last week only or throughout gestation, on cardiac output and uteroplacental blood flow in pregnant rats. J Nutr 113, 1766-1776

Barnes RJ, Comline RS, Dobson A (1986) The control of splanchnic blood flow. In: Control of digestion and metabolism in ruminants (Milligan LP, Grovum WL, Dobson A, eds) Prentice Hall, Englewood Cliffs NJ, 41-59

Beaton GH (1985) The significance of adaptation in the definition of nutrient requirements and for nutrition policy. In: Nutritional adaptation in man (Blaxter $\mathrm{K}$, Waterlow JC, eds) John Libbey, Londres, 219-232

Bell AW, Thompson GE (1979) Free fatty acid oxidation in bovine muscle in vivo: effects of cold exposure and feeding. Am I Physiol 237, E309-E315

Bergman EN, Wolff JE (1971) Metabolism of volatile fatty acids by liver and portal drained viscera in sheep. Am J Physiol 221, 586-592

Bird AR, Chandler KD, Bell AW (1981) Effects of exercise and plane of nutrition on nutrient utilization by the hind limb of the sheep. Austr $J$ Biol Sci 34, 541-550

Burrin DG, Britton RA, Ferrell CL (1988) Visceral organ size and hepatocyte metabolic activity in fed and fasted rats. $J$ Nutr $118,1547-1552$

Burrin DG, Ferrell CL, Britton RA, Bauer $M$ (1990) Level of nutrition and visceral organ size and metabolic activity in sheep. $B r J$ Nutr 64, 439-448

Burrin DG, Ferrell CL, Eisemann JH, Britton RA, Nienaber JA (1989) Effect of level of nutrition on splanchnic blood flow and oxygen consumption in sheep. Br J Nutr 62, 23-34

Canas R, Romero JJ, Baldwin RL (1982) Maintenance energy requirements during lactation in rats. $J$ Nutr 112, 1876-1880

Chérel Y, Le Maho Y (1985) Five months of fasting in king penguin chicks: body mass loss and fuel metabolism. Am J Physiol 249, R387-R392 
Chilliard Y, Rémond B, Agabriel J, Robelin J, Vérité $R$ (1987) Variations du contenu digestif et des réserves corporelles au cours du cycle gestation-lactation. Bull Tech CRZV Theix, INRA 70, 117-131

Chou CC (1983) Contribution of splanchnic circulation to overall cardiovascular and metabolic homeostasis. Fed Proc 42, 1656-1657

Chou CC, Gallavan RH (1982) Blood flow and intestinal motility. Fed Proc 41, 2090-2095

Christopherson RJ, Brockman RP (1989) Effect of feeding and a beta-adrenergic blocking agent on oxygen consumption by the portaldrained viscera, liver and hind quarters of the sheep. In: Energy metabolism of farm animals (van der Honing $Y$, Close $W H$, eds) Pudoc, Wageningen, 147-150

Clapperton JL, Blaxter KL (1965) Absence of long term adaptation in the energy metabolism of sheep on constant feed. Proc Nutr Soc 24, XXXIII-XXXIV

Coulson RA (1986) Metabolic rate and the flow theory: a study in chemical engineering. Comp Biochem Physiol 84A, 217-229

Doreau M, Robelin J, Lestrade A (1985) Effects of physiological state and body fatness on digestive tract weight and composition in the dairy cow. Livest Prod Sci 12, 379-385

Early RJ, Thompson JR, Christopherson RJ, Sedgwick GW (1984) Branched-chain alpha keto acid exchange across the portal-drained viscera and hindlimb of fed and fasted steers. Can J Anim Sci 64 (suppl) 276-277

Early RJ, Thompson JR, Christopherson RJ, Sedgwick GW (1987) Blood branched-chain amino acid and alpha keto acid concentrations and net exchange across the portaldrained viscera and hindlimb of fed and fasted ruminants. Can $J$ Anim Sci 67, 10111020

Edelstone DI, Holzman IR (1981) Gastrointestinal tract $\mathrm{O}_{2}$ uptake and regional blood flows during digestion in conscious newborn lambs. Am J Physiol 241, G289-G293

Eisemann JH, Nienaber JA (1989) Tissue and whole body oxygen uptake in fed and fasted steers. J Anim Sci 67 (suppl 1), 582

Eisemann JH, Nienaber JA (1990) Tissue and whole body oxygen uptake in fed and fasted steers. Br J Nutr 64, 399-411

Es AJH van (1972) Maintenance. In: Handbuch der Tierernahrung. Zweiter band. Leistungen und Ernahrung (Lenkeit W, Breirem K, Crasemann $E$, eds), Verlag Paul Parey, Hambourg et Berlin, 1-54

Farrell DJ, Leng RA, Corbett JL (1972) Undernutrition in grazing sheep. Il. Calorimetric measurements on sheep taken from pasture. Austr J Agric Res 23, 499-509

Ferrell CL, Jenkins TG (1984) A note on energy requirements for maintenance of lean and fat Angus, Hereford and Simmental cows. Anim Prod 39, 305-309

Ferrell CL, Koong LJ (1985) Response of body organs of lambs to differing nutritional treatments. In: Energy metabolism of farm animals (Moe PE, Tyrrell HF, Reynolds PJ, eds) Rowman \& Littlefield, NJ, 26-29

Ferrell $\mathrm{CL}$, Koong KJ (1986) Influence of plane of nutrition on body composition, organ size and energy utilization of Sprague-Dawley rats. J Nutr 116, 2525-2536

Ferrell CL, Koong LJ, Nienaber JA (1986) Effect of previous nutrition on body composition and maintenance energy costs of growing lambs. Br J Nutr 56, 595-605

Frisch JE, Vercoe JE (1977) Food intake, eating rate, weight gains, metabolic rate and efficiency of food utilization in Bos Taurus and Bos Indicus crossbred cattle. Anim Prod 25, 343-358

Gill M, France J, Summers M, McBride BW, Milligan LP (1989) Mathematical integration of protein metabolism in growing lambs. $J$ Nutr 119, 1269-1286

Gingins M, Bickel H, Schurch A (1980) Efficiency of energy utilization in undernourished and realimented sheep. Livest Prod Sci 7, 465471

Goodman MN, Larsen PR, Kaplan MM, Aoki TT, Young VR, Ruderman NB (1980) Starvation in the rat. II. Effect of age and obesity on protein sparing and fuel metabolism. Am J Physiol 239, E277-E286

Graham NMc (1964) Energetic efficiency of fattening sheep. II. Effects of undernutrition. Austr J Agric Res 15, 113-126

Graham NMc (1967) The metabolic rate of fasting sheep in relation to total and lean body weight, and the estimation of maintenance requirements. Austr J Agric Res 18, 127-136

Graham NMc (1969) The influence of body weight (fatness) on the energetic efficiency of adult sheep. Austr J Agric Res 20, 375-385 
Graham NMc, Searle TW, Griffiths DA (1974) Basal metabolic rate in lambs and young sheep. Austr J Agric Res 25, 957-971

Granger DN, Perry MA, Kvietys PR (1983) The microcirculation and fluid transport in digestive organes. Fed Proc 42, 1667-1672

Gregory NG, Christopherson RJ (1986) Effect of fasting on capillary blood flow in sheep. Res Vet Sci 40, 357-360

Guyton AC (1976) Medical physiology. WB Saunders Co, Philadelphia

Harris PM, Garlick PJ, Lobley GE (1989) Interactions between energy and protein metabolism in the whole body and hind limb of sheep in response to intake. In: Energy metabolism of farm animals (van der Honing $Y$, Close WH, eds) Pudoc, Wageningen, 167170

Heitman RN, Bergman EN (1981) Glutamate interconversions and glucogenicity in the sheep. Am J Physiol 241, E465-E472

Heitman RN, Sensenig SC, Reynolds CK, Fernandez JM, Dawes DJ (1986) Changes in energy metabolite and regulatory hormone concentrations and net fluxes across splanchnic and peripheral tissues in fed and progressively fasted ewes. J Nutr 116, 25162524

Hight GK, Barton RA (1965) The effects of undernutrition and realimentation on the Romney ewe. J Agric Sci 64, 413-424

Hochachka PW, Somero GN (1984) Biochemical Adaptation. Princeton Univ Press, Princeton NJ

Hohenboken WD, Hausser ER, Chapman AB, Cundiff LV (1972) Partitioning lactation TDN consumption in Herefords between maintenance, gain and milk production. J Anim Sci $34,152-160$

Huntington GB (1984) Relationship of portal blood flow to metabolizable energy intake of cattle. Can J Anim Sci 64 (suppl 1), 16-17

Huntington GB, Prior RL (1983) Digestion and absorption of nutrients by beef heifers fed a high concentrate diet. J Nutr 113, 2280-2288

Huntington GB, Prior RL, Britton RA (1980) Glucose and lactate absorption and metabolic interrelationships in lambs switched from low to high concentrate diets. J Nutr 110, 19041913
Huntington GB, Prior RL, Britton RA (1981) Glucose and lactate absorption and metabolic interrelationships in steers changed from low to high concentrate diets. J Nutr 111, 11641172

Huntington GB, Reynolds CK (1987) Oxygen consumption and metabolite flux of bovine portal-drained viscera and liver. J Nutr 117 , 1167-1173

Huntington GB, Reynolds PJ, Tyrrell HF (1983) Net absorption and ruminal concentrations of metabolites in non-pregnant dry Holstein cows before and after intraruminal acetic acid infusion. J Dairy Sci 66, 1901-1908

Huntington GB, Tyrrell HF (1985) Oxygen consumption by portal-drained viscera of cattle: comparison of analytical methods and relationship to whole body oxygen consumption. J Dairy Sci 68, 2727-2731

Huntington GB, Varga GA, Glenn BP, Waldo DR (1988) Net absorption and oxygen consumption by Holstein steers fed alfalfa hay or orchardgrass silage at two equalized intakes. J Anim Sci 66, 1292-1302

Jarrett IG, Filsell OH, Ballard FJ (1976) Utilization of oxidizable substrates by the sheep hind-limb: effects of starvation and exercise. Metabolism 25, 523-531

Jenkins TG, Ferrell CL, Cundiff LV (1986) Relationship of components of the body among mature cows as related to size, lactation potential and possible effects on productivity. Anim Prod 43, 245-254

Johnson CL, Johnson DE, Rumpler WV (1985) Source and level of alimentation effects on visceral organ mass of fat steers. In: Energy metabolism of farm animals (Moe PE, Tyrrell HF, Reynolds PJ, eds) Rowman \& Littlefield, NJ, 50-53

Johnson DE (1984) Maintenance requirements for beef cattle: importance and physiological and environmental causes of variation. In: Beef cow efficiency forum, East Lansing, MI, 6-14

Johnson DE, Johnson KA, Baldwin RL (1990) Changes in liver and gastro-intestinal tract energy demands in response to physiological workload in Ruminants. J Nutr 120, 649-655

Kaufman GL, Jr (1982) Blood flow and gastric secretion. Fed Proc 41, 2080-2083 
Keenan DM, McManus WR, Freer M (1969) Changes in the body composition and efficiency of mature sheep during loss and regain of live weight. J Agric Sci (Camb) 72, 139-147

Kelly JM, Vaage AS, McBride BW, Milligan LP (1989) Oxygen consumption and the energy costs of $\mathrm{Na}^{+}, \mathrm{K}^{+}$ATPase in rumen epithelial papillae from Hereford steers. J Anim Sci 67 (suppl 1), 560

Klosterman EW, Sanford LG, Parker CF (1968) Effect of cow size and condition and ration protein content upon maintenance requirements of mature beef cows. J Anim Sci 27, 242-246

Koong LJ, Ferrell CL, Nienaber JA (1982) Effects of plane of nutrition on organ size and fasting heat production in swine and sheep. In: Energy metabolism of farm animals (Ekern A, Sundstol F, eds) Agric Univ Norway, Áas, 245-248

Koong LJ, Ferrell CL, Nienaber JA (1985) Assessment of interrelationships among levels of intake and production, organ size and fasting heat production in growing animals. $J$ Nutr 115, 1383-1390

Koong LJ, Nienaber JA (1985) Changes of fasting heat production and organ size of pigs during prolonged weight maintenance. In: Energy metabolism in farm animals (Moe PW, Tyrell HF, Reynolds PJ, eds) Rowman \& Littlefield, Totowa New Jersey

Lautt WW (1983) Relationship between hepatic blood flow and overall metabolism: the hepatic arterial buffer response. Fed Proc 42, 1662-1666

Leche TF (1973) Proportions of carcass and offal components in Jersey and Friesian bulls in relation to plane of nutrition. Austr $J$ Agric Res 24, 623-631

Lederman SA, Resso P (1989) The pattern of plasma volume changes in well nourished and in food or protein restricted pregnant rats. J Am Coll Nutr 8, 215-224

Ledger HP, Sayers AR (1977) The utilization of dietary energy by steers during periods of restricted food intake and subsequent realimentation. I. The effect of time on the maintenance requirements of steers held at constant live weights. J Agric Sci (Camb) 88, 1126
Lipinski HG (1989) Model calculations of oxygen supply to tissue slice preparations. Phys Med Biol 34, 1103-1111

Lomax MA, Baird GD (1983) Blood flow and nutrient exchange across the liver and gut of the dairy cow. Effects of lactation and fasting. Br J Nutr 49, 481-496

Ma SWY, Foster DO (1986) Starvation-induced changes in metabolic rate, blood flow and regional energy expenditure in rats. Can J Physiol Pharmacol 64, 1252-1258

Mailman D (1982) Blood flow and intestinal absorption. Fed Proc 41, 2096-2100

Marston HR (1948) Energy transactions in the sheep. I. The basal heat production and heat increment. Austr J Sci Res 1, 93-129

McBride BW, Milligan LP (1982) Energy expenditure associated with $\mathrm{Na}^{+}$and $\mathrm{K}^{+}$transport in intestinal epithelium of sheep and cattle. Can J Anim Sci 62, 1263

McBride BW, Milligan LP (1984) The effect of lactation on ouabain-sensitive respiration of the duodenal mucosa of cows. Can $J$ Anim Sci 64, 817-824

McBride BW, Milligan LP (1985) Influence of feed intake and starvation on the magnitude of $\mathrm{Na}^{+}, \mathrm{K}^{+}$-ATPase (EC 3.6.1.3.)-dependent respiration in duodenal mucosa of sheep. $\mathrm{Br}$ $\checkmark$ Nutr 53, 605-614

McBride BW, Early RJ, Ball RO (1989) Protein synthesis and the energy costs of $\mathrm{Na}^{+}, \mathrm{K}^{+}$ transport in tissues of somatotropin treated steers. In: Energy metabolism of farm animals (van der Honing $\mathrm{Y}$, Close $\mathrm{WH}$, eds) Pudoc, Wageningen, 107-110

McNiven MA (1984) The effect of body fatness on energetic efficiency and fasting heat production in adult sheep. Br J Nutr 51, 297-304

Meyer JH, Clawson WJ (1964) Undernutrition and subsequent realimentation in rats and sheep. J Anim Sci 23, 214-224

O'Donovan PB (1984) Compensatory gain in cattle and sheep. Nutr Abstr Rev B 54, 389410

Parks JR (1982) $A$ theory of feeding and growth of animals. Springer Verlag, Berlin

Pethick DW, Harman N, Chong JK (1987) Nonesterified long chain fatty acid metabolism in fed sheep at rest and during exercise. Austr $J$ Biol Sci 40, 221-234 
Pethick DW, Lindsay DB, Barker PJ, Northrop AJ (1981) Acetate supply and utilization by the tissues of sheep in vivo. Br J Nutr 46, 97110

Petit M (1988) Alimentation des vaches allaitantes. In: Alimentation des bovins, ovins et caprins (Jarrige R, ed), INRA, Paris, 159-184

Petit $M$, Micol $D$ (1981) Evaluation of energy requirements of beef cows during early lactation. Livest Prod Sci 8, 139-153

Prior RL, Huntington GB, Britton RA (1981) Influence of diet on amino acid absorption in beef cattle and sheep. $J$ Nutr 111, 2212-2222

Reynolds CK, Tyrrell HF (1987) Effect of diet intake level on net visceral tissue metabolism in growing beef heifers. J Anim Sci 65 (suppl 1), 477

Reynolds CK, Tyrrell HF (1989) Effects of forage to concentrate ratio and intake on visceral tissue and whole body energy metabolism of growing beef heifers. In: Energy metabolism of farm animals (van der Honing $Y$, Close WH, eds) Pudoc, Wageningen, 151154

Robelin J, Agabriel J, Malterre C, Bonnemaire J (1990) Changes in body composition of mature dry cows of Holstein, Limousin and Charolais breeds during fattening. I. Skeleton, muscles, fatty tissues and offal. Livest Prod Sci 25, 199-215

Robin JP, Frain M, Sardet C, Groscolas R, Le Maho $Y$ (1988) Protein and lipid utilization during long-term fasting in emperor penguins. Am J Physiol 254, R61-R68

Rompala RE, Hoagland TA (1987) Effect of level of alimentation on visceral organ mass and the morphology and $\mathrm{Na}^{+}, \mathrm{K}^{+}$adenosinetriphosphatase activity of intestinal mucosa in lambs. J Anim Sci 65, 1058-1063

Rompala RE, Hoagland TA, Meister JA (1988) Effect of dietary bulk on organ mass, fasting heat production and metabolism of the small and large intestines in sheep. J Nutr 118, 1553-1557

Rompala RE, Johnson DE, Rumpler WV, Phetteplace HW, Parker CF (1987) Level of alimentation and line of breeding on oxygen uptake by ovine jejunal mucosa. Am J Physiol 252, R398-R401

Russel AJF, Wright IA (1983) Factors affecting maintenance requirements of beef cows. Anim Prod 37, 329-334
Sakanashi TM, Brigham HE, Rasmussen KM (1987) Effect of dietary restriction during lactation on cardiac output, organ blood flow and organ weights of rats. $J$ Nutr 117,1469 1474

Schneider W, Siems W, Grune T (1990) Balancing of energy consuming processes of rat hepatocytes. Cell Biochem Funct 8, 227-232

Seebeck RM (1967) Developmental growth and body weight loss of cattle. I. Experimental design, body weight growth, and the effects of developmental growth and body weight loss on the dressed carcass and the offal. Austr $J$ Agric Res 18, 1015-1031

Shepherd AP (1982) Metabolic control of intestinal oxygenation and blood flow. Fed Proc 41, 2084-2089

Silanikove $N$ (1987) Effect of imposed reduction of energy intake on resting and fasting heat production in the black bedouin desert goat. Nutr Rep int 35, 725-731

Summers $M$, McBride BW, Milligan LP (1988) Components of basal energy expenditure. In: Aspects of digestive physiology in ruminants (Dobson A, Dobson MJ, eds) Cornell Univ Press, ithaca NJ, 257-282

Thompson WR, Meiske JC, Goodrich RD, Rust JR, Byers FM (1983) Influence of body composition on energy requirements of beef cows during winter. $J$ Anim Sci 56, 12411252

Turner HG, Taylor CS (1983) Dynamic factors in models of energy utilization with particular reference to maintenance requirement of cattle. World Rev Nutr Diet 42, 135-190

Umbreit WW, Burris RH, Stauffer JF (1964) Manometric technics. Burgess Publi Co, Minneapolis Minnesota

Varga GA, Tyrrell HF (1989) Effect of prior rate of gain and end weight on energy metabolism, visceral organ mass and body composition of Angus $x$ Hereford steers. In: Energy metabolism of farm animals (van der Honing Y, Close WH, eds) Pudoc, Wageningen, 287290

Wagner JJ, Lusby KS, Oltjen JW, Rakestraw J, Wettemann RP, Walters LE (1988) Carcass composition in mature Hereford cows: estimation and effect on daily metabolizable energy requirement during winter. J Anim Sci $66,603-612$ 
Waldo DR, Tyrell HF (1980) The relation of insoluble nitrogen intake to gain, energy retention and nitrogen retention in Hostein steers. In: Protein metabolism and nutrition (Oslage HJ, Rohr K, eds), Braunschweig, 572-581

Waterlow JC (1985) What do we mean by adaptation? In: Nutritional adaptation in man (Blaxter K, Waterlow JC, eds) John Libbey, Londres, 1-10
Webster AJF (1981) The energetic efficiency of metabolism. Proc Nutr Soc 40, 121-128

Webster AJF, Osuji PO, White F, Ingram JF (1975) The influence of food intake on portal blood flow and heat production in the digestive tract of sheep. Br J Nutr 34, 125-139

Wieghart $M$, Slepetis $R$, Elliot JM, Smith DF (1986) Glucose absorption and hepatic gluconeogenesis in dairy cows fed diets varying in forage content. J Nutr 116, 839-850 